\title{
Fabrication and Study of Coupled Silicon Microring CROWs (Project Report 1028553)
}

\section{SHAYAN MOOKHERJEA}

Department of Electrical and Computer Engineering, University of California, San Diego, MC 0407 La Jolla CA 92093-0407 USA

Email:smookher@ucsd.edu

\section{Introduction and Goals}

The goal of this NSF-funded "GOALI" supplement project [1028553] is to theoretically and experimentally study (at UCSD) silicon photonic circuits fabricated (at IBM) in order to understand the effects of, and develop mitigation strategies for fabrication disorder. A collaboration with IBM's silicon photonics group provided insights into silicon photonics fabrication as relevant for industry. The project supported in-person technical collaboration on designing, fabricating and measuring silicon photonic structures. Several jointly-authored publications resulted from this collaboration [1]-[3]. CROWs of up to 235 coupled resonators were successfully fabricated and measured, and a good agreement was achieved between experiments and theory [4]-[6].

The coupled-resonator optical waveguide (CROW) structure that was the foundation for most of our joint studies in this GOALI project can have many

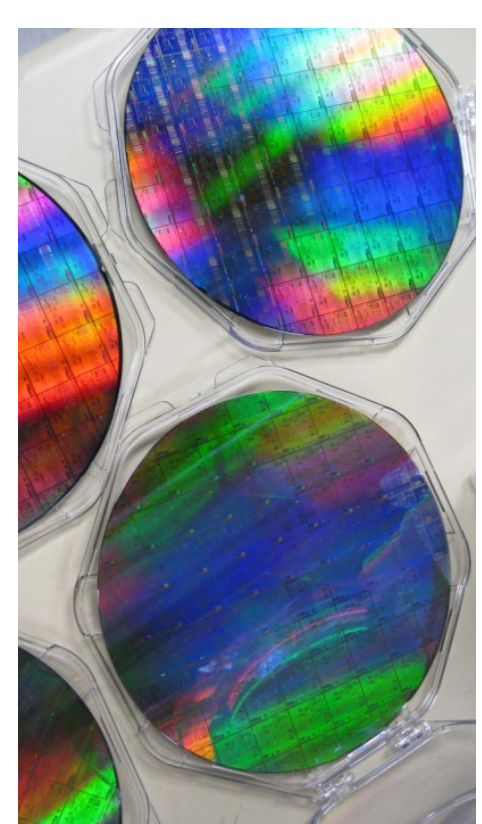

Figure 1 CROW structures fabricated as part of silicon photonic wafers. potential applications including low-power nonlinear optics [7], [8], novel lasers [9] and generation of entangled photon pairs through spontaneous four-wave mixing (SFWM) [10]. Many of these properties are related to the nontraditional dispersion characteristics of CROWs [11]. Although tight-binding models of bandcenter propagation exist [12]-[14], studying optical propagation in the regions of high dispersion [15] and high nonlinearity is difficult because of the sensitivity to disorder [16], [17]. In the main project (0925133), optical transport measurements were made and were compared to earlier theoretical models [2], [18].

\section{Activities}

A variety of CROW structures ranging from 35 to 235 coupled rings were experimentally studied, achieving a new benchmark in the development 
of CROWs [19]. An SEM image of a 35-ring CROW is shown in Fig. 1. By measuring light transmission versus the length of the structure, a loss-per-ring of $0.135 \mathrm{~dB}$ per resonator was inferred. As a comparison, CROWs using coupled polymer rings had shown $2.8 \mathrm{~dB}$ per ring loss [20]. An image of light at a specific wavelength near $1550 \mathrm{~nm}$ propagating through the 35-ring CROW structure is shown in Figure 3.

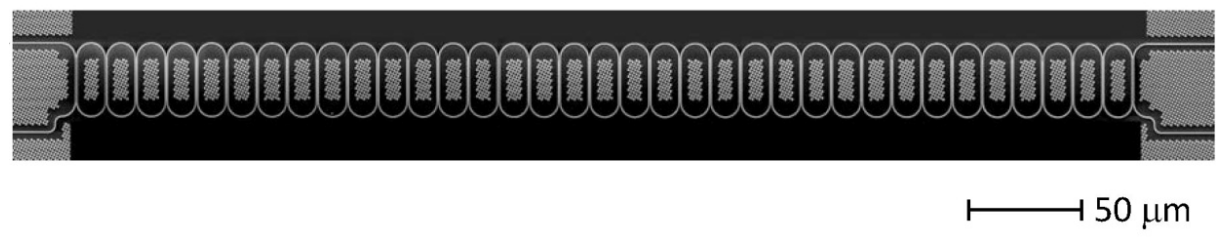

Figure 2 Scanning electron microscope (SEM) image of a CROW with 35 coupled ring resonators in silicon photonics, fabricated by IBM. The raw image has been edited in software to digitally remove the fill pattern outside the rings (similar to the fill pattern seen inside the rings), for visual clarity.

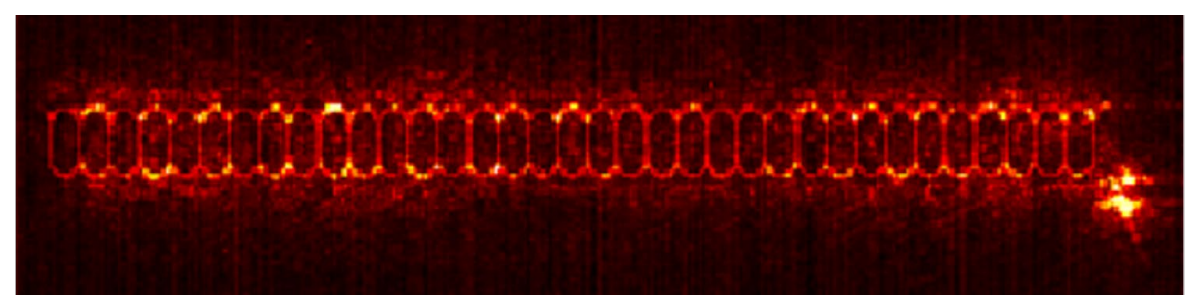

Figure 3 Infrared camera image of light at a wavelength near $1550 \mathrm{~nm}$ propagating through the 35 ring CROW. The image was taken using a InGaAs infrared camera $(320 \times 256$ pixel resolution) and a microscope with a high magnification objective. The input is to the bottom right hand side of this image and the light propagates from right to left.

Infrared imaging is a useful experimental tool to study light propagation through the length of these structures [21], [22]. Images such as shown in Figure 3 were collected at many wavelengths across a selected passband of the CROW. The image was integrated over each ring's circumference to obtain an excitation amplitude (of optical intensity). Figure 3 shows the spatially-resolved distribution of excitation amplitudes from the input to the output ports of the CROW as a function of optical wavelength (CW excitation). The measured optical transmission is superimposed using a blue trace (and numerical values using the right-hand side vertical axis). This clearly shows that extended "supermodes" which are collective resonances of all resonators spatially span the entire length of the $\mathrm{CROW}$ structure. By integrating along the racetrack circumference of each resonator, an excitation amplitude is calculated for each unit cell. The sequence of excitation amplitudes of the unit cells from input to output thus depict the magnitude of the CROW eigenmode at a particular collective resonance frequency (eigenvalue). Note that the inter-resonator phase cannot be obtained in this manner. 
We developed an extension of coupled-mode theory which is applicable to directional couplers in silicon photonics, coupled-waveguide structures [23] as well as coupled-resonator structures [24]. The CROW structures used in these experiments are based on microring resonators with directional couplers. Previously, we have experimentally studied the dispersion of such couplers using a microring coupled to a waveguide [25]. An accurate knowledge of dispersion is also necessary for planar lightwave circuits which use CROWs (as filters) together with other devices [26]. To experimentally study CROWs with different dispersion characteristics from a single wafer fabrication run, the exposure properties of the photolithography process was graded across the wafer from north to south (see Figure 4). This resulted in different gaps and waveguide widths which were compared with simulations in order to calculate the resulting coupling coefficients.

\section{0 mm SOI Wafer (100) Surface}

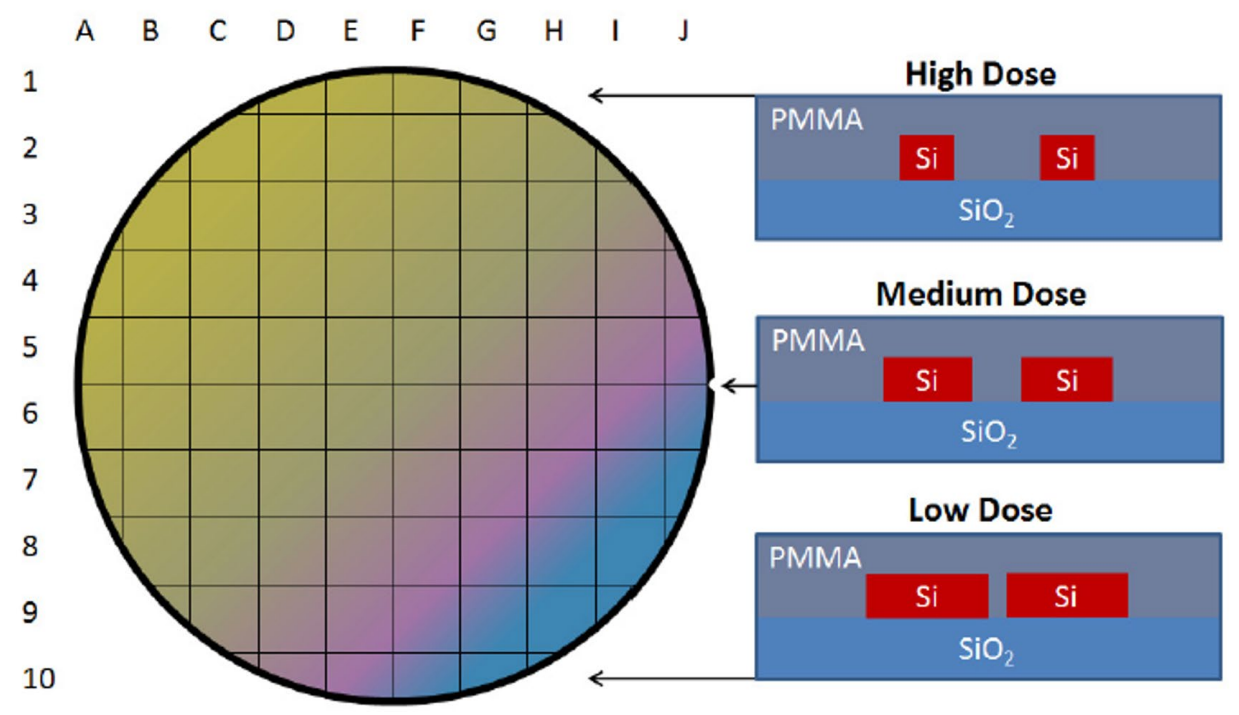

Figure 4 Grading the photolithography exposure dose resulted in changing the waveguide widths and gaps across the wafer, which yielded microchips with different waveguiding properties based on the same design file.

In practice, we need a way to dynamically tune the CROW waveguiding properties. In silicon photonics, directly-pumped optical gain is not available [27]. In this project, we used electro-optic tunability because of its practical convenience. Using off-the-shelf electronics equipment such as arbitrary waveform generators, it was easy to generate the switching waveforms required in our experiments [28]. As later research showed, electronic control over optical propagation can be implemented using the electro-optic effect even at 
speeds less than $10 \mathrm{ps}$ [29]. If tuning speed is not a concern, the thermo-optic effect, which we used for tunable filters, is also another option as a control knob [30] but thermo-optic heaters were not included as part of these fabricated structures. Alternatively, optical pump beams can be used to manipulate the waveguiding properties through nonlinear optical properties. This can be difficult to control in practice since silicon microresonators can be affected by some phenomena that are not always desirable for CROWs, such as bistability [31].

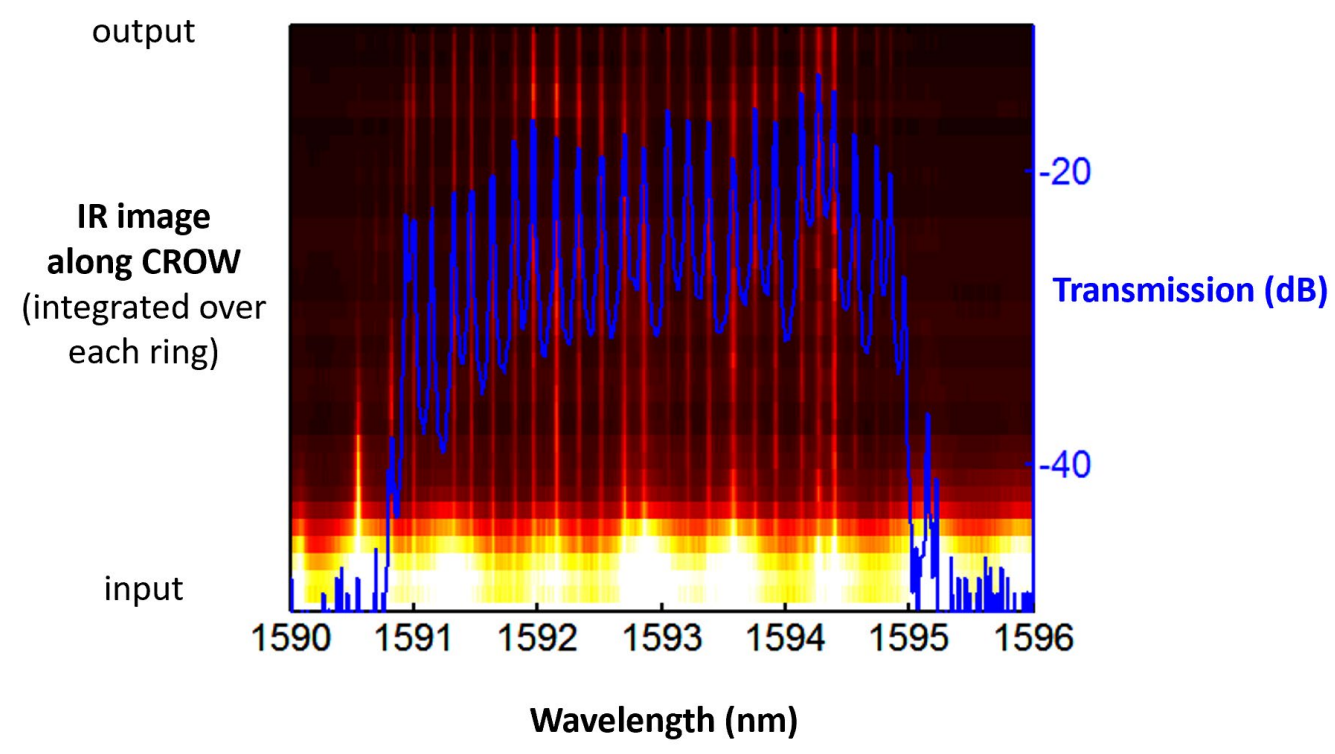

Figure 5 Wavelength-resolved and spatially-resolved infrared camera imaging of the supermodes which comprise the passband transmission. As the input wavelength is (step-) scanned across the passband, infrared images were acquired of light propagation along the length of the CROW. Raw images were processed in software by path-integrating the infrared intensity around the perimeter of each microring. The resulting amplitudes (magnitude only) are plotted along the vertical axis, and depict the CROW eigenmodes.

\section{Progress and Impact}

The PI spent two months at the IBM Watson Research Center (July and August, 2009) during which time CROWs were fabricated by IBM based on joint design and discussions. The benefit to the university is the access to fabrication design tools and resources of scale and ability far exceeding that of any in-house university fabrication facility. This potentially leads to deeper scientific insights, better diagnostic methodologies, and improved measurement procedures. The benefit to the industrial partner is the collaborative investigation of high-risk ideas and accomplishing more time-consuming and labor-intensive basic-research studies in silicon photonics. A joint study agreement was negotiated between the entities, which stated as our mutual goal that "the mechanisms leading to degraded performance in practical structures, 
including fabrication-induced disorder ... will be studied in an effort to improve the fundamental performance of slow light devices".

CROWs shown in Fig. 2 were fabricated at the IBM Microelectronics Research Laboratory on $200 \mathrm{~mm}$ silicon-on-insulator wafers $(220 \mathrm{~nm}$ thick silicon layer, $2 \mu \mathrm{m}$ thick oxide layer). Each segment of the CROW shown in Fig. 2 consists of a microring resonator, formed in the racetrack configuration (bending radius $6.5 \mu \mathrm{m}$, straight section approximately $20 \mu \mathrm{m}$ ) using silicon nanowire waveguides. The waveguides were single-mode, with transverse dimensions approximately $0.50 \mu \mathrm{m} \times 0.20 \mu \mathrm{m}$, which is sufficient to strip the transversemagnetic polarization of light after a few bends. The inter-resonator coupling gaps vary from $150 \mathrm{~nm}$ (apodized input section, which consists of 3 unit cells each at the input and output sections) to $270 \mathrm{~nm}$ (the remaining section of the waveguide), achieving the over-coupled regime of waveguide-ring coupling. This was achieved using the processing steps shown in Figure 4.

The waveguides fabricated in this process were fully-etched silicon "strip" waveguides, which typically have a propagation loss of $6-8 \mathrm{~dB} / \mathrm{cm}$ at 1550 $\mathrm{nm}$ at the time of this project. Additional processing steps were used to reduce the optical propagation loss. A double oxidation process was partially successful at reducing the propagation loss. Two oxidation steps were used, each oxidizing about $10 \mathrm{~nm}$ of silicon to silicon dioxide. The first layer of silicon dioxide was removed with a wet etch dip before the second oxidation step was performed. The oxide from the second step was left on the waveguide perimeter. A cladding of PMMA was then spin-coated over the wafer. The lowest propagation loss was achieved using a high-temperature forming gas annealing step carried out on the wafer during the fabrication process to smoothen the sidewalls (see Figure 6). A low-index polymer $(n=1.48)$ was used as the upper cladding.

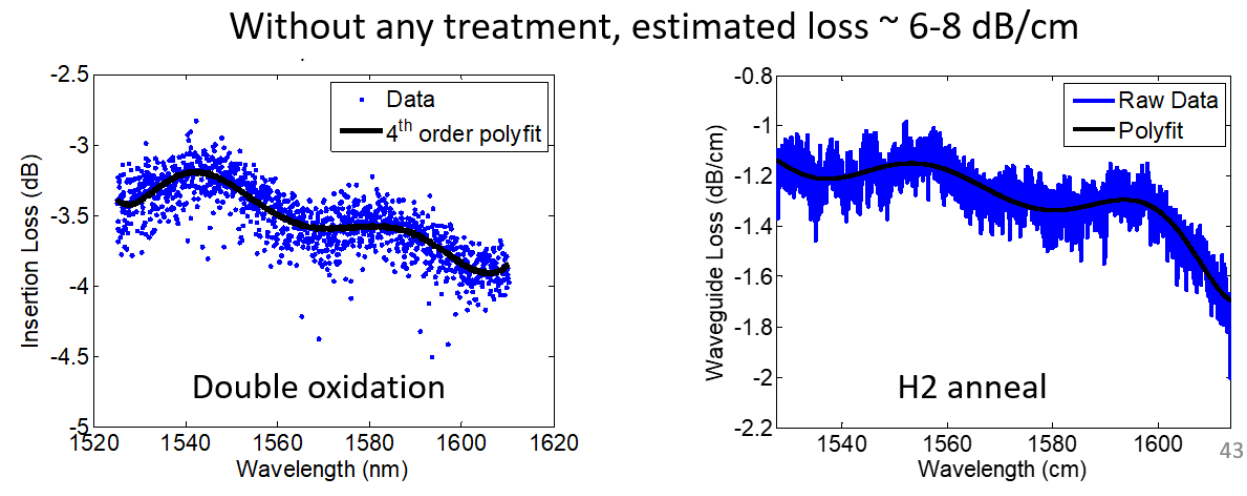

Figure 6 Comparison of silicon waveguide propagation loss after (left panel) two steps of oxidation of a few nanometers and (right panel) a forming gas anneal, which slightly rounds off the corners of the waveguide and smoothens the rough edges. 
Additional external collaborations: Dr. Ivan B. Divliansky (Research Scientist, CREOL, Florida) collaborated with us for the electron-beam lithography of long waveguide structures. Jointly-authored publications resulted from this collaboration [21]. Novel measurement techniques were developed based on a single-scan, Jones matrix-based, interferometric spectral measurement instrument (Luna Tech. Optical Vector Analyzer OVA5000, generously loaned by Luna Technologies, VA. A jointly-authored publication resulted from this collaboration [2].
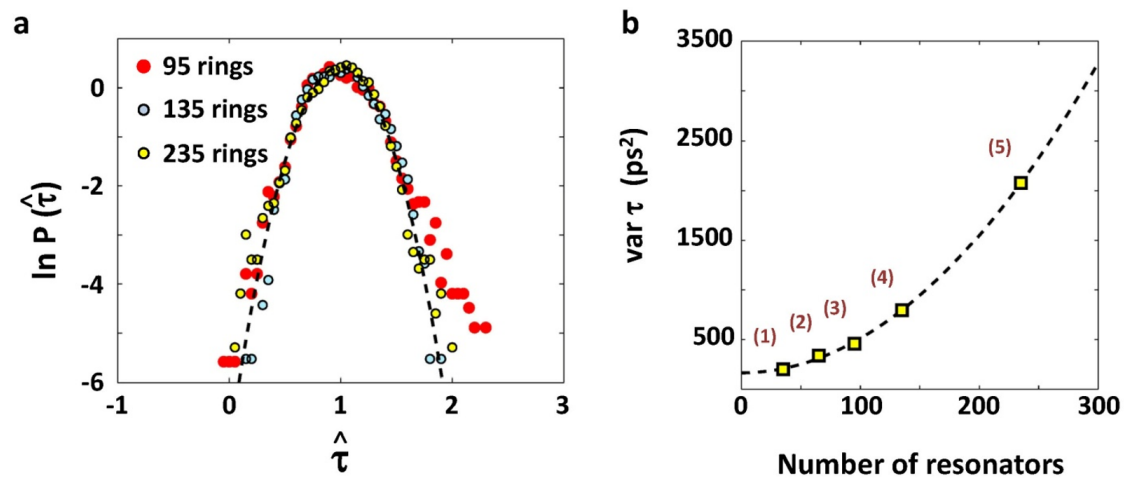

In any uncorrelated-increments random process, the variance increases as $\mathrm{L}$.

In a correlated-increments random process, the variance increases as $\mathrm{L}^{2}$.

Figure 7 Scaling behavior of group delay ripple. Measurements of optical transmission in the passband across different lengths of waveguide (different number of rings) were analyzed statistically. The variance of the normalized group delay was seen to scale quadratically with the number of resonators (or device length), indicating a correlated-increments random process.

\section{Subsequent Extensions}

From the experimental observations made in this project (see Fig. 6), we inferred that a $\mathrm{CROW}$ introduces strong spectral correlations in propagating photons and that these correlations are robust to both loss and disorder. Therefore, it seemed likely that a nonlinear optical process such as spontaneous four-wave mixing would be well suited to generating photon pairs with strong quantum correlations. This was indeed verified experimentally soon afterwards. Efficient FWM is possible in single microrings [32] as is pair generation through spontaneous four-wave mixing (SFWM) [33]. However, one of the unique characteristics of the CROW is the ability to vary the two-photon state in ways not possible using a single microring resonator [34].

We continue to study and develop both the single-resonator and coupledresonator configurations for quantum light generation using spontaneous fourwave mixing [35]. The resonator-enhanced nonlinearity would benefit not only 
classical nonlinear optics such as second-harmonic generation (SHG) [36] but also the generation of entangled photon-pair and heralded single-photon generation using silicon photonics [10], [37]. Like single microrings, CROWs can generate photon pairs across multiple bands (spectrally separated by the free spectral range, FSR) and the center wavelength of the bands can be thermally tuned [38]. A single microresonator can be monitored and controlled more easily [39] than a long CROW device and an optimized single silicon microresonator can also be useful for nonlinear wavelength conversion with low pump powers in the milliwatt range [32]. However, an efficient secondorder nonlinear (chi-2) waveguide can also generate photon pairs with pump powers in the milliwatt range [40], and a more detailed comparison is needed to assess the relative merits of the two approaches.

If the silicon surface can be modified after fabrication, device performance may be improved by reconfiguring silicon lightwave circuits to compensate the effect of disorder. We demonstrated this functionality using some devices whose fabrication was supported by this project. The method we used is based on field-induced local oxidation of $\mathrm{Si}$ to $\mathrm{SiO}_{2}$ via a chemical reaction near an electrically-biased conducting atomic-force microscope tip [41], [42]. This is a site-specific oxidation method, with nanoscale lateral and vertical resolution, and introduces no new materials or process requirements. Reconfiguration of the light path through a Mach-Zehnder interferometer (switching between cross and bar parts) and shifting of the resonance of a microring resonator were both demonstrated experimentally.

The optical Kerr nonlinearity which is used as the physics principle for resonator-enhanced FWM and SFWM can also be used in other contexts to confine light in small physical region, e.g., in spatial solitons [43], [44]. The CROW and other coupled optical structures can support solitons in both the spatial and temporal domains [45], [46].

\section{Open-Access Reporting Initiative}

PRAISE: This open-access document is provided in support of our PRAISE (Public Report of Activities, Impact and Subsequent Extensions) initiative. What is it? An open-access document shared with the public which describes the research outcomes of publicly-funded projects. For us, these projects are typically funded by the NSF (National Science Foundation).

\section{References}


[1] M. L. Cooper et al., "Statistics of light transport in 235-ring silicon coupled-resonator optical waveguides," Opt. Express, vol. 18, no. 25, p. 26505, Dec. 2010, doi: 10.1364/OE.18.026505.

[2] M. L. Cooper et al., "Waveguide dispersion effects in silicon-on-insulator coupled-resonator optical waveguides," Opt. Lett., vol. 35, no. 18, p. 3030, Sep. 2010, doi: 10.1364/OL.35.003030.

[3] J. R. Ong et al., "Low-power continuous-wave four-wave mixing in silicon coupled-resonator optical waveguides," Opt. Lett., vol. 36, no. 15, pp. 2964-2966, 2011.

[4] M. L. Cooper et al., "235-ring Coupled-Resonator Optical Waveguides," in Conference on Lasers and Electro-Optics 2010, San Jose, California, 2010, p. CTuHH3. doi: 10.1364/CLEO.2010.CTuHH3.

[5] S. Mookherjea and M. A. Schneider, "Avoiding bandwidth collapse in long chains of coupled optical microresonators," Opt. Lett., vol. 36, no. 23, p. 4557, Dec. 2011, doi: 10.1364/OL.36.004557.

[6] M. L. Cooper and S. Mookherjea, "Modeling of Multiband Transmission in Long Silicon Coupled-Resonator Optical Waveguides," IEEE Photon. Technol. Lett., vol. 23, no. 13, pp. 872-874, Jul. 2011, doi: 10.1109/LPT.2011.2141657.

[7] S. Mookherjea and A. Yariv, "Kerr-stabilized super-resonant modes in coupled-resonator optical waveguides," Phys. Rev. E, vol. 66, no. 4, p. 046610, Oct. 2002, doi: 10.1103/PhysRevE.66.046610.

[8] S. Mookherjea and A. Yariv, "Optical pulse propagation and holographic storage in a coupled-resonator optical waveguide," Phys. Rev. E, vol. 64, no. 6, p. 066602, Nov. 2001, doi: 10.1103/PhysRevE.64.066602.

[9] S. Mookherjea, "Semiconductor coupled-resonator optical waveguide laser," Appl. Phys. Lett., vol. 84, no. 17, pp. 3265-3267, Apr. 2004, doi: 10.1063/1.1719278.

[10] R. Kumar, M. Savanier, J. R. Ong, and S. Mookherjea, "Entanglement measurement of a coupled silicon microring photon pair source," Opt.

Express, vol. 23, no. 15, p. 19318, Jul. 2015, doi: 10.1364/OE.23.019318.

[11] S. Mookherjea, "Dispersion characteristics of coupled-resonator optical waveguides," Opt. Lett., vol. 30, no. 18, p. 2406, Sep. 2005, doi: 10.1364/OL.30.002406.

[12] S. Mookherjea and A. Yariv, "Optical pulse propagation in the tightbinding approximation," Opt. Express, vol. 9, no. 2, p. 91, Jul. 2001, doi: 10.1364/OE.9.000091.

[13] N. Stefanou and A. Modinos, "Impurity bands in photonic insulators," Phys. Rev. B, vol. 57, no. 19, pp. 12127-12133, May 1998, doi:

10.1103/PhysRevB.57.12127.

[14] S. Olivier et al., "Miniband transmission in a photonic crystal coupledresonator optical waveguide," Opt. Lett., vol. 26, no. 13, pp. 1019-1021, 2001. 
[15] W. J. Kim, W. Kuang, and J. D. O’Brien, "Dispersion characteristics of photonic crystal coupled resonator optical waveguides," Opt. Express, vol. 11, pp. 3431-3437, 2003.

[16] S. Mookherjea, D. S. Cohen, and A. Yariv, "Nonlinear dispersion in a coupled-resonator optical waveguide," Opt. Lett., vol. 27, no. 11, p. 933, Jun. 2002, doi: 10.1364/OL.27.000933.

[17] S. Mookherjea and A. Oh, "Effect of disorder on slow light velocity in optical slow-wave structures," Opt. Lett., vol. 32, no. 3, p. 289, Feb. 2007, doi: 10.1364/OL.32.000289.

[18] S. Mookherjea and A. Yariv, "Pulse propagation in a coupled resonator optical waveguide to all orders of dispersion," Phys. Rev. E, vol. 65, no. 5, p. 056601, Apr. 2002, doi: 10.1103/PhysRevE.65.056601.

[19] F. Morichetti, C. Ferrari, A. Canciamilla, and A. Melloni, "The first decade of coupled resonator optical waveguides: bringing slow light to applications," Laser \& Photon. Rev., vol. 6, no. 1, pp. 74-96, Jan. 2012, doi: 10.1002/lpor.201100018.

[20] J. K. Poon, L. Zhu, G. A. DeRose, and A. Yariv, "Transmission and group delay of microring coupled-resonator optical waveguides," Opt. Lett., vol. 31, no. 4, p. 456, Feb. 2006, doi: 10.1364/OL.31.000456.

[21] M. L. Cooper, G. Gupta, J. S. Park, M. A. Schneider, I. B. Divliansky, and S. Mookherjea, "Quantitative infrared imaging of silicon-on-insulator microring resonators," Opt. Lett., vol. 35, no. 5, p. 784, Mar. 2010, doi: 10.1364/OL.35.000784.

[22] S. Mookherjea and H. R. Grant, "High dynamic range microscope infrared imaging of silicon nanophotonic devices," Opt. Lett., vol. 37, no. 22, p. 4705, Nov. 2012, doi: 10.1364/OL.37.004705.

[23] M. L. Cooper and S. Mookherjea, "Numerically-assisted coupledmode theory for silicon waveguide couplers and arrayed waveguides," Opt. Express, vol. 17, no. 3, p. 1583, Feb. 2009, doi: 10.1364/OE.17.001583.

[24] S. Mookherjea, "Spectral characteristics of coupled resonators," $J$. Opt. Soc. Am. B, vol. 23, no. 6, p. 1137, Jun. 2006, doi: 10.1364/JOSAB.23.001137.

[25] R. Aguinaldo, Yiran Shen, and S. Mookherjea, "Large Dispersion of Silicon Directional Couplers Obtained via Wideband Microring Parametric Characterization," IEEE Photon. Technol. Lett., vol. 24, no. 14, pp. 1242-1244, Jul. 2012, doi: 10.1109/LPT.2012.2198639.

[26] J. R. Ong, R. Kumar, and S. Mookherjea, "Silicon microring-based wavelength converter with integrated pump and signal suppression," Opt. Lett., vol. 39, no. 15, p. 4439, Aug. 2014, doi: 10.1364/OL.39.004439.

[27] S. Mookherjea, "Using gain to tune the dispersion relation of coupledresonator optical waveguides," IEEE Photon. Technol. Lett., vol. 18, no. 5, pp. 715-717, Mar. 2006, doi: 10.1109/LPT.2006.871144. 
[28] S. Mookherjea, J. R. Ong, X. Luo, and L. Guo-Qiang, "Electronic control of optical Anderson localization modes," Nature Nanotech, vol. 9, no. 5, pp. 365-371, May 2014, doi: 10.1038/nnano.2014.53.

[29] X. Wang, P. O. Weigel, J. Zhao, M. Ruesing, and S. Mookherjea, "Achieving beyond-100-GHz large-signal modulation bandwidth in hybrid silicon photonics Mach Zehnder modulators using thin film lithium niobate," APL Photonics, vol. 4, no. 9, p. 096101, Sep. 2019, doi: 10.1063/1.5115243.

[30] R. Aguinaldo et al., "Wideband silicon-photonic thermo-optic switch in a wavelength-division multiplexed ring network," Opt. Express, vol. 22, no. 7, p. 8205, Apr. 2014, doi: 10.1364/OE.22.008205.

[31] S. Mookherjea and M. A. Schneider, "The nonlinear microring adddrop filter," Opt. Express, vol. 16, no. 19, p. 15130, Sep. 2008, doi: 10.1364/OE.16.015130.

[32] J. R. Ong, R. Kumar, R. Aguinaldo, and S. Mookherjea, "Efficient CW Four-Wave Mixing in Silicon-on-Insulator Micro-Rings With Active Carrier Removal," IEEE Photon. Technol. Lett., vol. 25, no. 17, pp. 1699 1702, Sep. 2013, doi: 10.1109/LPT.2013.2272521.

[33] C. Ma, X. Wang, V. Anant, A. D. Beyer, M. D. Shaw, and S. Mookherjea, "Silicon photonic entangled photon-pair and heralded single photon generation with CAR $>12,000$ and $\mathrm{g}^{\wedge}(2)(0)<0006$, , Opt. Express, vol. 25, no. 26, p. 32995, Dec. 2017, doi: 10.1364/OE.25.032995.

[34] R. Kumar, J. R. Ong, M. Savanier, and S. Mookherjea, "Controlling the spectrum of photons generated on a silicon nanophotonic chip," Nat Commun, vol. 5, no. 1, p. 5489, Dec. 2014, doi: 10.1038/ncomms6489.

[35] J. R. Ong and S. Mookherjea, "Quantum light generation on a silicon chip using waveguides and resonators," Opt. Express, vol. 21, no. 4, p. 5171, Feb. 2013, doi: 10.1364/OE.21.005171.

[36] S. Mookherjea and A. Yariv, "Second-harmonic generation with pulses in a coupled-resonator optical waveguide," Phys. Rev. E, vol. 65, no. 2, p. 026607, Jan. 2002, doi: 10.1103/PhysRevE.65.026607.

[37] M. Davanço et al., "Telecommunications-band heralded single photons from a silicon nanophotonic chip," Appl. Phys. Lett., vol. 100, no. 26, p. 261104, Jun. 2012, doi: 10.1063/1.4711253.

[38] R. Kumar, J. R. Ong, J. Recchio, K. Srinivasan, and S. Mookherjea, "Spectrally multiplexed and tunable-wavelength photon pairs at $155 \mu \mathrm{m}$ from a silicon coupled-resonator optical waveguide," Opt. Lett., vol. 38, no. 16, p. 2969, Aug. 2013, doi: 10.1364/OL.38.002969.

[39] M. Savanier, R. Kumar, and S. Mookherjea, "Optimizing photon-pair generation electronically using a $p-i-n$ diode incorporated in a silicon microring resonator," Appl. Phys. Lett., vol. 107, no. 13, p. 131101, Sep. 2015, doi: 10.1063/1.4932047. 
[40] J. Zhao, C. Ma, M. Rüsing, and S. Mookherjea, "High Quality Entangled Photon Pair Generation in Periodically Poled Thin-Film Lithium Niobate Waveguides," Phys. Rev. Lett., vol. 124, no. 16, p. 163603, Apr. 2020, doi: 10.1103/PhysRevLett.124.163603.

[41] Y. Shen, I. B. Divliansky, D. N. Basov, and S. Mookherjea, "Electricfield-driven nano-oxidation trimming of silicon microrings and interferometers," Opt. Lett., vol. 36, no. 14, p. 2668, Jul. 2011, doi: 10.1364/OL.36.002668.

[42] Y. Shen, I. B. Divliansky, D. N. Basov, and S. Mookherjea, "Perfect set-and-forget alignment of silicon photonic resonators and interferometers," in Optical Fiber Communication Conference/National Fiber Optic Engineers Conference 2011, Los Angeles, California, 2011, p. PDPC3. doi: 10.1364/OFC.2011.PDPC3.

[43] A. Ciattoni, B. Crosignani, S. Mookherjea, and A. Yariv, "Nonparaxial dark solitons in optical Kerr media," Opt. Lett., vol. 30, no. 5, p. 516, Mar. 2005, doi: 10.1364/OL.30.000516.

[44] B. Crosignani, A. Yariv, and S. Mookherjea, "Nonparaxial spatial solitons and propagation-invariant pattern solutions in optical Kerr media," Opt. Lett., vol. 29, no. 11, p. 1254, Jun. 2004, doi: 10.1364/OL.29.001254.

[45] D. N. Christodoulides and N. K. Efremidis, "Discrete temporal solitons along a chain of nonlinear coupled microcavities embedded in photonic crystals," Opt. Lett., vol. 27, no. 8, pp. 568-570, 2002.

[46] J. E. Heebner, R. W. Boyd, and Q.-H. Park, "SCISSOR solitons and other novel propagation effects in microresonator-modified waveguides," J. Opt. Soc. Am. B, vol. 19, pp. 722-731, 2004. 